\title{
ON WEAK MIXING METRIC AUTOMORPHISMS ${ }^{1}$
}

\author{
BY JAMES W. ENGLAND ${ }^{2}$ AND N. F. G. MARTIN
}

Communicated by P. R. Halmos, November 27, 1967

Let $(X, Q, P)$ be a separable probability space and $T$ a metric automorphism of the space onto itself, i.e. $T$ is, except for null sets, a one to one invertible map from $X$ to $X$ such that $T^{-1}(a)=a$ and $P\left(T^{-1} A\right)=P(A)$ for all $A \in Q$.

There are three standard types of mixing for metric automorphisms, namely ergodic, weak mixing, and strong mixing [2]. It is known [1] that an automorphism is ergodic if and only if for all sets $A$ and $B$ from $Q$ which have nonzero measure there exists a positive integer $n$ such that $P\left(T^{n} A \cap B\right)>0$. In this note we show that a similar condition which we call property $\mathrm{W}$ is necessary and sufficient for weak mixing.

Property W. For every two sets $A$ and $B$ of strictly positive measure there exists a subset $K$ of the positive integers with density zero such that for all $k \notin K, P\left(T^{k} A \cap B\right)>0$.

Lemma. If a metric automorphism $T$ satisfies property $\mathrm{W}$ then it is strongly ergodic, i.e. every nonzero integral power of $T$ is ergodic.

Proof. Let $m$ be a given positive integer and $A$ and $B$ two sets of positive measure. Let $K$ denote the set of density zero associated with $A$ and $B$ by property W. Denote by $M$ the set of integers $m k$ where $k$ runs over the positive integers. Since the upper density of $M$ is positive, $M$ is not contained in $K$ and there exists $m k \notin K$. Thus $P\left(T^{m k} A \cap B\right)=P\left(\left(T^{m}\right)^{k} A \cap B\right)>0$ and $T^{m}$ is ergodic. Since $T$ ergodic implies $T^{-1}$ ergodic, $T^{m}$ is ergodic for all nonzero integers.

THEOREM. A necessary and sufficient condition that a metric automorphism $T$ be weak mixing is that it have property $\mathrm{W}$.

Proof. Suppose first $T$ is weak mixing. Then (see [2]) for $A$ and $B$ given sets of nonzero measure, there exists a subset $K^{\prime}$ of integers with density zero such that $\lim _{n \notin K^{\prime}} P\left(T^{n} A \cap B\right)=P(A) P(B)>0$. Thus for all $n$ not in $K^{\prime}$ and larger than some integer $N, P\left(T^{n} A \cap B\right)$ $>0$. Let $K=K^{\prime} \cup\{k: 0 \leqq k \leqq N, k$ integer $\}$. The set $K$ has density zero and if $n \notin K$ then $P\left(T^{n} A \cap B\right)>0$.

1 This work was supported in part by ARO Grant 662 .

2 National Science Foundation Fellow. 
Next suppose $T$ has property $\mathrm{W}$. Then $T$ is ergodic and hence the induced unitary operator on $L_{2}(P)$ defined by $\left(U_{T f}\right)(x)=f(T x)$ has simple eigenvalues and eigenfunctions with constant absolute values (see [2]). Suppose $T$ is not weak mixing. Then $U_{T}$ has an eigenvalue $\lambda \neq 1$ (see [2]). Let $f_{0}$ denote the eigenfunction associated with $\lambda$ such that $\left|f_{0}\right|=1$. Since $\lambda$ is not 1 and $T$ is ergodic, $f_{0}$ is nonconstant. Also since every power of $T$ is ergodic, $\lambda$ cannot be a root of unity.

Let $S$ denote the circle and $R_{\lambda}$ the $\lambda$-rotation on $S$, i.e. $R_{\lambda} z=\lambda z$ for all $z \in S$. Since $\left|f_{0}\right|=1$, the range of $f_{0}$, which we shall denote by $Y$, is a subset of $S$ and by direct computation one may show that $Y$ is invariant under $R_{\lambda}$. If $T_{\lambda}$ denotes the restriction of $R_{\lambda}$ to $Y$, then $T_{\lambda}$ is a one to one map of $Y$ onto $Y$.

Let $B$ denote the Borel subsets of $S$ and $\mathcal{C}$ denote the $\sigma$-algebra $B \cap Y=\{B \cap Y: B \in B\}$ of subsets of $Y$. Since $f_{0}^{-1}(B \cap Y)=f_{0}^{-1}(B)$ and $f_{0}$ is a measurable function, $f_{0}^{-1}(\mathcal{C}) \subset Q$. Thus $f_{0}$ is a measurable function on $(X, \mathfrak{Q})$ onto $(Y, \mathbb{C})$. Likewise since $T_{\lambda}^{-1}(B \cap Y)=R_{\lambda}^{-1}(B) \cap Y$ and $R_{\lambda}$ is a Borel map, $T_{\lambda}$ is a measurable function on $(Y, \mathrm{e})$ onto $(Y, \mathrm{e})$.

Define the probability $P_{*}$ on $(Y, \mathfrak{e})$ by $P_{*}(A \cap Y)=P f_{0}^{-1}(A)$. If $A \cap Y \in \mathrm{e}$, then

$$
P_{*}\left[T_{\lambda}^{-1}(A \cap Y)\right]=P f_{0}^{-1}\left[R_{\lambda}^{-1}(\mathrm{~A}) \cap Y\right]=P f_{0}^{-1}\left[R_{\lambda}^{-1}(A)\right] .
$$

Since $T$ is measure preserving $P_{*}(A \cap Y)=P\left(T^{-1}\left(f_{0}^{-1} A\right)\right)$. However, $f_{0}^{-1}\left(R_{\lambda}^{-1} A\right)=T^{-1}\left(f_{0}^{-1} A\right)$ so that $P_{*}(A \cap Y)=P_{*}\left[T_{\lambda}^{-1}(A \cap Y)\right]$ and $T_{\lambda}$ is $P_{*}$-measure preserving. Thus $\left(Y, \mathfrak{e}, P_{*}\right)$ is a probability space and $T_{\lambda}$ is a metric automorphism of the space. Moreover $f_{0}$ is a metric homomorphism of $(X, Q, P)$ onto $\left(Y, \mathcal{e}, P_{*}\right)$ such that $f_{0} T=T_{\lambda} f_{0}$. That is, the dynamical system $(X, Q, P, T)$ is homomorphic to the dynamical system $\left(Y, \mathfrak{e}, P_{*}, T_{\lambda}\right)$.

Now we show that since $T$ satisfies property $\mathrm{W}$ and $T_{\lambda}$ is a homomorphic image of $T, T_{\lambda}$ must satisfy property W. Let $A$ and $B$ be two sets from $\mathcal{C}$ which have positive $P_{*}$-measure and denote $f_{0}^{-1}(A)$ and $f_{0}^{-1}(B)$ respectively by $A^{\prime}$ and $B^{\prime}$. Since these two sets have positive $P$-measure and $T$ has property $\mathrm{W}$ there is the set $K$ of density zero associated with $A^{\prime}$ and $B^{\prime}$ by property $\mathrm{W}$. Let $n$ be a positive integer not in $K$. Since $f_{0}^{-1}\left(T_{\lambda}^{n} A\right) \cap f_{0}^{-1}(B) \supset T_{\lambda}^{n}\left(f_{0}^{-1}(A)\right) \cap f_{0}^{-1}(B)$ we have that

$$
\begin{aligned}
P_{*}\left(T_{\lambda}^{n} A \cap B\right) & =P\left[f_{0}^{-1}\left(T_{\lambda}^{n} A\right) \cap f_{0}^{-1}(B)\right] \\
& \geqq P\left(T^{n} A^{\prime} \cap B^{\prime}\right)>0
\end{aligned}
$$

and $T_{\lambda}$ satisfies property $\mathrm{W}$. 
However, since $T_{\lambda}$ is a certain restriction of a rotation on the circle we can show that it cannot satisfy property $\mathrm{W}$ and this contradiction will complete the proof.

Suppose there exists a nondegenerate arc $I_{0}$ in $S$ such that $P_{*}\left(I_{0} \cap Y\right)=0$. Since $\lambda$ is not a root of unity, $R_{\lambda}$ is an irrational rotation on the circle and hence $\cup_{n=0}^{\infty} R_{\lambda}^{n} I_{0}=S$. Thus

$$
1=P_{*}(Y)=P_{*}\left[\bigcup_{n=0}^{\infty} T_{\lambda}^{n}\left(I_{0} \cap Y\right)\right] \leqq \sum_{n=0}^{\infty} P_{*}\left(T_{\lambda}^{n}\left(I_{0} \cap Y\right)\right)=0
$$

and this contradiction implies that for every nondegenerate arc $I$ of $S, P_{*}(I \cap Y)>0$.

Now, let $I_{0}$ denote the arc of $S$ between 1 and $i$ and $I_{1}$ denote the arc of $S$ between -1 and $-i$. Since an irrational rotation is uniformly almost periodic there exists a relatively dense set $\left\{n_{i}\right\}$ of integers such that $R_{\lambda}^{n_{i}} I_{0} \cap I_{0} \neq \varnothing$. But this implies that $R_{\lambda}^{n_{i}} I_{0} \cap I_{1}=\varnothing$ and it follows that $T_{\lambda}^{n_{i}}\left(I_{0} \cap Y\right) \cap\left(I_{1} \cap Y\right)=\varnothing$ for all $i$. Since $I_{0} \cap Y$ and $I_{1} \cap Y$ have positive $P_{*}$-measure if $T_{\lambda}$ satisfied condition $\mathrm{W}$, then the set $\left\{n_{i}\right\}$ would have to be a subset of a set of density zero and hence itself have density zero. This is impossible since $\left\{n_{i}\right\}$ is relatively dense.

It might be of some interest to point out that the theorem together with the lemma shows that weak mixing automorphisms are strongly ergodic. Also in the proof of the theorem a homomorphic image of a nonweak mixing automorphism was constructed which was a rotation on an invariant subset of the circle. Since this implies that nonweak mixing automorphisms have factor automorphisms with zero entropy we have that an automorphism with completely positive entropy must be weak mixing. However, it is known [3] that automorphisms with completely positive entropy are mixing of all orders.

Finally, we conjecture that a necessary and sufficient condition that a metric automorphism $T$ be strongly mixing is that for every two sets $A$ and $B$ of nonzero measure there exists an integer $N$ such that for all $n \geqq N, P\left(T^{n} A \cap B\right)>0$.

\section{BIBLIOGRAPHY}

1. Nathaniel Friedman, Introduction to Ergodic theory, Mimeo. Lecture Note, University of New Mexico, Albuquerque, 1966.

2. Paul R. Halmos, Leciures on ergodic theory, Chelsea, New York, 1956.

3. V. A. Rohlin, New progress in the theory of transformations with invariant measure, Uspeki Math. Nauk 15 (1960), 3-26=Russian Math. Surveys 15 (1960), 1-22.

UNIVERSITY OF VIRGINIA AND

INSTITUTE FOR AdVANCEd StUdy 